\title{
IMPLEMENTATION OF MULTI-WAVELENGTH SOURCE FOR DWDM-PON FIBER OPTICAL TRANSMISSION SYSTEMS
}

\author{
K. Vilcane ${ }^{1 *}$, S. Matsenko ${ }^{1,2}$, M. Parfjonovs ${ }^{1}$, R. Murnieks ${ }^{1,2}$, \\ M. Aleksejeva ${ }^{1,2}$, S. Spolitis ${ }^{1,2}$ \\ 'Institute of Telecommunications, Riga Technical University, \\ 12 Azenes Str., LV-1048, Riga, LATVIA \\ ${ }^{2}$ Communication Technologies Research Center, Riga Technical University, \\ 12 Azenes Str., LV-1048, Riga, LATVIA \\ *e-mail: Klinta.Vilcane1@rtu.Iv
}

Four-wave mixing (FWM) is one of the well-known nonlinear optical effects (NOE), and it is considered as an adverse impact in fibre optical communication lines. This nonlinear optical effect as a productive one can be used in fibre optical communication systems for various optical processing functions, like wavelength conversion, high-speed time-division multiplexing (TDM), pulse compression, fibre optical parametric amplifiers (FOPA), etc. In most of the fibre optical communication systems, each data transmission channel requires one light source (e.g., laser) as a carrier, which can make these transmission systems expensive. For example, to provide operation of 4-channel dense wavelength-division-multiplexed (DWDM) system four separate lasers at specific operation wavelengths are needed. On the contrary, through the FWM effect, which can be obtained in highly nonlinear optical fibre (HNLF) by using two high-power pump lasers, the generation of new multiple carriers forming the laser array or a multi-wavelength source is possible. Accordingly, within the present research, we investigate the latter approach for FWM light source implementation in DWDM passive optical networks (DWDM-PONs). We analyse up to 16-channel $50 \mathrm{GHz}$ spaced DWDM-PON system with a bitrate of up to $10 \mathrm{Gbit} / \mathrm{s}$ per channel, constructed on the basis of two high-power continuous wave $(\mathrm{CW})$ pump lasers. We evaluate the system performance against the number of its channels by changing it from 4 to 16 and in each case find the most optimal HNLF fibre length (for a 4-channel system it is $0.9 \mathrm{~km}$; for an 8-channel system - $1.39 \mathrm{~km}$; and for a 16-channel system $-1.05 \mathrm{~km}$ ) and laser pump powers (for a 4-channel system it is $20 \mathrm{dBm}$; for an 8-channel 
system $-24.1 \mathrm{dBm}$; and for a 16-channel system $-26.3 \mathrm{dBm})$. These optimal parameters were found in order to get the highest system performance, respectively, the lowest BER (threshold BER $\leq 10^{-10}$ ), and minimal power fluctuations among FWM generated carriers. The obtained results show that the proposed transmission system can be a promising solution for next-generation high-speed PONs.

Keywords: dense wavelength division multiplexed passive optical network (DWDM$P O N)$, four-wave mixing (FWM), highly nonlinear optical fiber (HNLF), nonlinear optical effects (NOE).

\section{INTRODUCTION}

An increase in the amount of information transmitted and an ever-growing number of Internet users have increased the demand for network bandwidth and further transition to new architectures and data transmission technologies. High spectral efficiency dense wavelength-divisionmultiplexed (DWDM) systems are used to increase the rapidly growing demand for the Internet traffic, using multimedia applications, streaming video, file sharing, etc. [1]. Dense wavelength-division-multiplexed passive optical network (DWDMPON) is compatible with fibre-to-the-home (FTTH) architecture by providing flexibility and far greater capacity. DWDM fibre optical transmission system technology is used for long-distance data transmission; however, DWDM signals are exposed by nonlinear optical effects (NOE). In the process of data transmission, interference, distortion, excessive attenuation of the optical signals may appear due to the nonlinearity of the optical fibre, which leads to a degradation in performance [2]. One of the most common nonlinear optical effects in optical fibre communication systems is Kerr nonlinearities - the influence of frequency modulation or pulse spectral components of the movement. Different effects such as self-phase modulation (SPM), cross-phase modulation (XPM), and four-wave mixing (FWM) occur due to the Kerr nonlinearity [3], [4]. Nonlinear FWM is an effect when two or more different signals with different frequencies propagate next to each other, and because of this effect, new carriers are generated. In addition, existing channels are exposed to power loss and signal distortions. The FWM effect can be used to generate multiple carriers. The FWM effect is adverse for DWDM-PON transmission systems, but nowadays solutions for useful application of this effect are being researched.

The application of the FWM optical effect was researched to create up to a 16-channel DWDM-PON system. FWM multi-wavelength source is based on two continuous wave (CW) lasers and optical highly nonlinear fibre (HNLF). In this article, we developed and analysed setups of up to a 16-channel DWDM-PON system, where optimal power of both pump CW lasers and HNLF fibre span length were determined. The performance of the received signals was analysed in terms of bit error ratio (BER), where we set that the BER threshold according to PON networks was $\mathrm{BER} \leq 10^{-10}$. 


\section{GENERATION OF FOUR-WAVE MIXING NONLINEAR OPTICAL EFFECT}

The four-wave mixing may occur in fibre optical systems as the intermodulation phenomenon in which the fourth wavelength arises due to an interaction among three wavelengths. If there are three wavelengths $\lambda_{i}, \lambda_{j}$, and $\lambda_{k}$, which are mixed, then it leads to the appearance of the fourth wavelength, as shown in formula 1 below [5]:

$$
\lambda_{i j k}=\lambda_{i} \pm \lambda_{j} \pm \lambda_{k}
$$

where $\lambda_{\mathrm{ijk}}$ - may act as an interfering signal to the original signal. The worst-case combination of these wavelengths degrades the performance of a system [5]. Three waves of wavelength $\lambda_{i}, \lambda_{j}$, and $\lambda_{k}(i, j \neq k)$ interact to generate a wave with wavelength [6]: $\lambda_{i j k}=\lambda_{i}+\lambda_{j}-\lambda_{k},(i, j \neq k)$,

where $\lambda_{\mathrm{ijk}}-$ the wavelength of the generated FWM signal.

Formula (3) describes how many new channels intend to be in a system. For $N$ wavelength channels co-propagating through the fibre, the number of new wavelengths that FMW effect generates is [7]:

$M=\frac{N^{2}}{2}(N-1)$.

Figure 1 shows the optical signal spectrum before and after transmission along the optical fibre with the impact of FWM. The power of the FWM signal is influenced by such factors as dispersion of the transmission fibre, channel input power and spectral spacing between the carriers or channels.
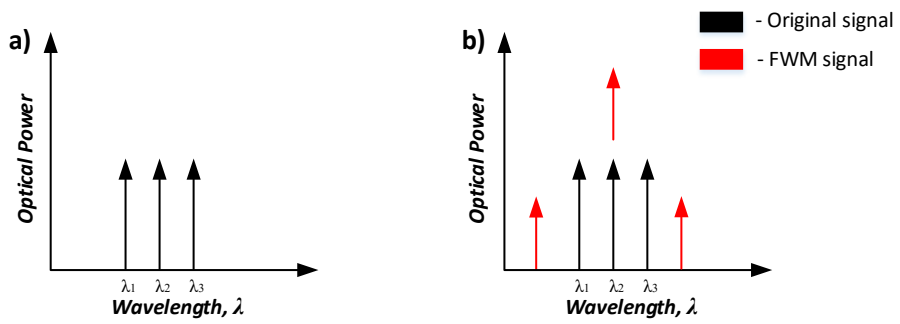

Fig. 1. The spectrum of optical signal a) without FWM and b) with the occurrence of the FWM effect [5].

\section{ARCHITECTURE OF DWDM-PON TRANSMISSION SYSTEM WITH FWM SOURCE}

DWDM-PON can be used as a multiple channel optical fibre transmission system to be able to serve as many customers as possible at the same time and gain higher power and bitrate [7], [8].

According to ITU-T G.694.1 recom- mendation for optical $\mathrm{C}$ and $\mathrm{L}$ bands, the DWDM-PON channel spacing intervals are $100,50,25$ or $12.5 \mathrm{GHz}$, and the frequency of the central channel is $193.1 \mathrm{THz}$ [9]. In this article, a DWDM-PON transmission system with channel spacing $50 \mathrm{GHz}$ is 
under research. This system uses an FWM multi-wavelength source to provide further information transmission to multiple data channels. Figure 2 shows the architecture of multichannel DWDM-PON transmission system.

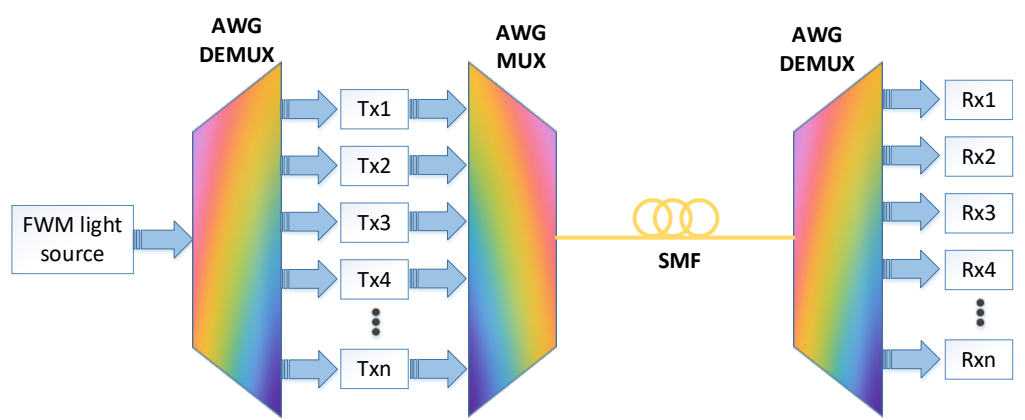

Fig. 2. The architecture of the multichannel DWDM-PON transmission system with FWM multi-wavelength source [10].

As it is shown in Fig. 2, the simulated transmission system employs one arrayedwaveguide grating (AWG) multiplexer (MUX) for the combination of modulated carriers and two demultiplexers (DEMUX) for filtering and separation of carriers generated by FWM multi-wavelength source (transmitter side) and separation of the received DWDM signals at the receiver side.

\section{EXPERIMENTAL SIMULATION MODEL AND NUMERICAL ANALYSIS}

In this article, the use of the FWM optical effect was researched to create a multiwavelength source for up to a 16-channel DWDM-PON system. We developed an experimental simulation model with an FWM multi-wavelength source for the DWDM-PON transmission system in the RSOFT OptSim simulation software environment. The power of pumping lasers, as it is shown in Fig. 4, was changed in the range from $+15 \mathrm{dBm}$ to $+30 \mathrm{dBm}$. Different HNLF fibre lengths up to $2 \mathrm{~km}$ were also studied [11].

As a result of the FWM process, the fourth frequency was generated. To efficiently generate nonlinear FWM in HNLF fibre, the following HNLF parameters were set: the core effective area of $11.6 \mu \mathrm{m}^{2}$, zero dispersion wavelength of $1552.32 \mathrm{~nm}$ and the nonlinear coefficient of $11.50(\mathrm{~W} \times \mathrm{km})^{-1}$ [11].
Central frequencies applied in this research for pump CW lasers were 193.10 $\mathrm{THz}(1552.524 \mathrm{~nm})$ and $193.15 \mathrm{THz}$ $(1552.123 \mathrm{~nm})$, attenuation coefficient for a generation of up to 16 carriers in HNLF fibre was $0.8 \mathrm{~dB} / \mathrm{km}$ at the reference wavelength of $1550 \mathrm{~nm}$ [11]. The parameters of AWG MUX/DEMUX employed in DWDM-PON systems were as follows: channel spacing was $50 \mathrm{GHz}$, optical 3-dB bandwidth was $16.5 \mathrm{GHz}$ and the lowest channel frequency of AWG MUX/DEMUX varied depending on the number of channels used: for 4 channels $-193.05 \mathrm{THz}$, for 8 channels -192.95 THz, for 16 channels $-192.75 \mathrm{THz}$. Table 1 shows optimal parameters for both $\mathrm{CW}$ lasers and HNLF fibre employed accordingly in 4-, 8- and 16-channel DWDMPON transmission systems. The optimal power of pumping CW lasers and HNLF fibre length were defined experimentally 
by using simulation software. These parameters were found by evaluating the optical spectrum (see Fig. 3) on the output of the first AWG demux, considering the lowest power variation of generated carriers, which did not exceed the range of $3 \mathrm{~dB}$.

Table 1. Optimal Parameters for the Generation of Multiple Carriers

\begin{tabular}{|c|c|c|}
\hline Number of carriers (channels) & CW pump laser power, $\mathrm{dBm}$ & HNLF length, $\mathrm{km}$ \\
\hline 4 & 20.0 & 0.90 \\
\hline 8 & 24.1 & 1.39 \\
\hline 16 & 26.3 & 1.05 \\
\hline
\end{tabular}

Figure 3 shows output spectra of FWM multi-wavelength source, where (a) 4-channel, (c) 8-channel, (e) 16-channel after the HNLF output and (b) 4-channel, (d) 8-channel, (f) 16-channel after the AWG multiplexer. Spectrum diagrams can be used to determine the quality of the selected parameters because the spectrum on the HNLF output will be smoother. Then it will be possible to find the lowest BER and maximum uniform carrier capacity for minimal fluctuations.

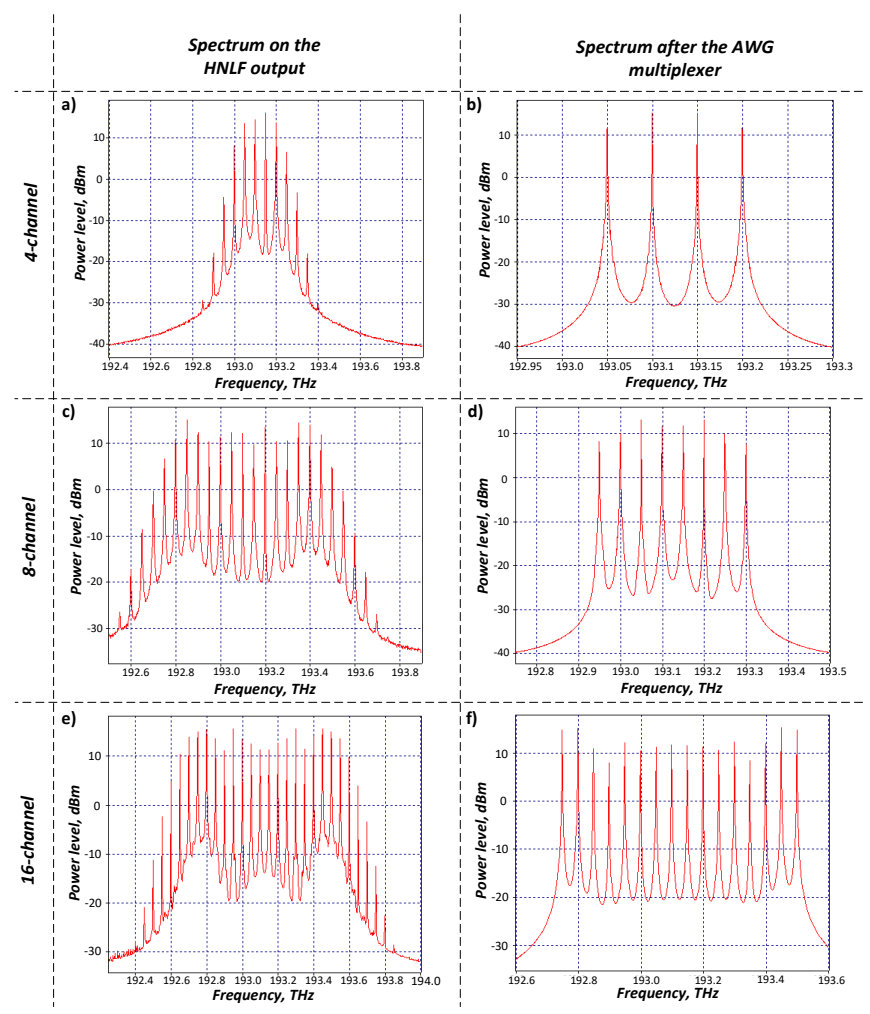

Fig. 3. The measured optical spectra (a, c, e) on the output of HNLF and (b, d, f) after AWG multiplexer at the transmission side of up to 16-channel DWDM-PON transmission system.

We configured the FWM multi-wavelength source, the parameters of which varied depending on the number of channels used in the system. Figure 4 shows the experimental simulation model of (a) up to 8-channel (including 4-channel) and (b) 16-channel DWDM-PON transmission system with the defined FWM multi-wavelength source. 


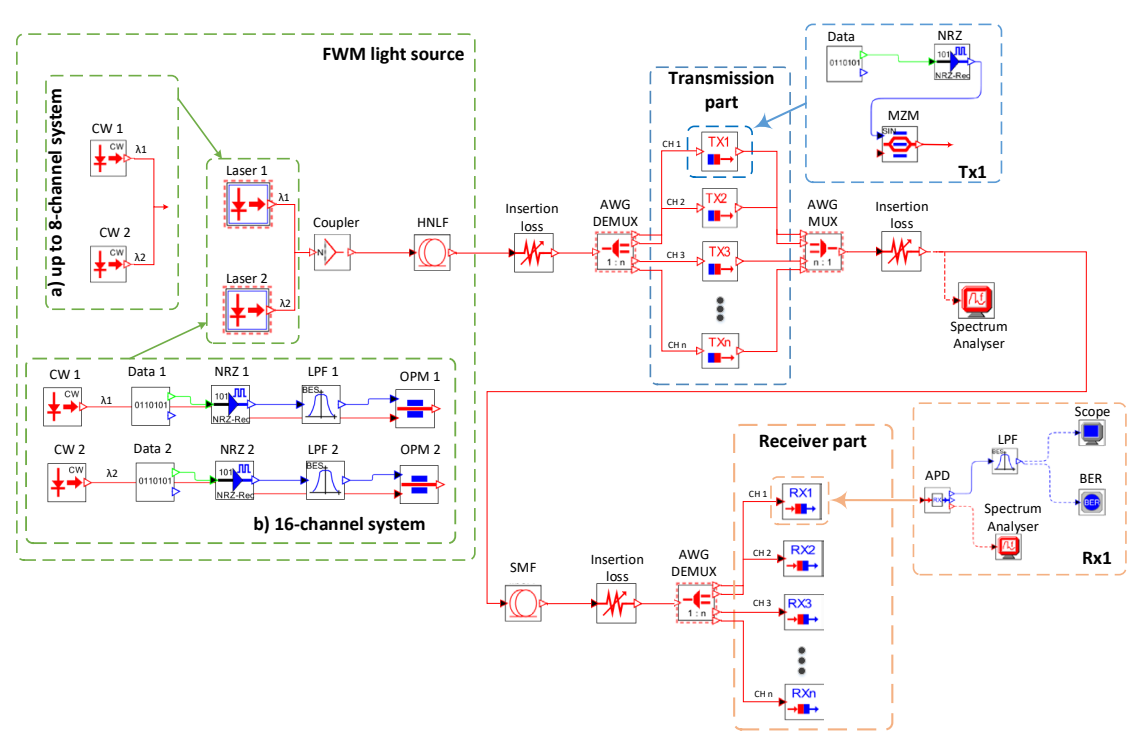

Fig. 4. Experimental simulation model of (a) 4 and 8-channel and (b) 16-channel DWDM-PON transmission system with an FWM multi-wavelength source.

In the DWDM-PON transmission system, some modifications were applied for multi-wavelength light source in the case of 16-carrier generation to obtain event distribution of the generated carrier powers. When the number of data channels increases, the carrier can be specially modulated to reduce the adverse effects of Brillouin scattering (SBS). An increase in the number of data channels increases the nonlinear Kerr effect as well as the stimulated Brillouin scattering [12]. In the case of SBS, energy is pumped from one light wave to another with a larger wavelength. SBS limits the maximum injected power (amplitude manipulation increases the width of the spectrum, as well as the SBS threshold), which is the reason why the use of more powerful lasers can be limited. To reduce SBS effect, the passive optical components with low attenuation are used [13], [14]. Two extra components - optical phase modulators (OPM) are used in multi-wavelength source scheme of the 16-channel system (see. Fig. 4 (b)) to increase SBS threshold and decrease the impact of SBS. Each of the pump laser sources is modulated by a bitrate of $100 \mathrm{Mbit} / \mathrm{s}$ [15] (non-return to zero (NRZ) line code, $2.8 \mathrm{GHz} 3-\mathrm{dB}$ bandwidth low-pass filters (LPF)).

In addition to the AWG MUX/DEMUX, an optical element was added, which simulated optical attenuation that introduced the required loss. This optical element in Fig. 4 is depicted as insertion loss - value is $3-\mathrm{dB}$. Data transmission channels with the 10 Gbit/s bitrate consist of data source (Data), NRZ coder and optical Mach-Zehnder modulator (MZM), where 3-dB bandwidth is $10 \mathrm{GHz}$ and excess loss is 3-dB. During the research, the number of data transmission channels varied from 4 to 16 channels. Optical ITU-T G.652 single-mode fibre (SMF) was used as transmission media; its length was $20 \mathrm{~km}$, the attenuation coefficient was $0.2 \mathrm{~dB} / \mathrm{km}$ and the dispersion coefficient was $16 \mathrm{ps} / \mathrm{nm} / \mathrm{km}$ at $1550 \mathrm{~nm}$ reference wavelength.

The receiver of the DWDM-PON system was formed of a sensitivity optical receiver where avalanche photodiode (ADP) was used with sensitivity of $-24 \mathrm{dBm}$ 
at reference error probability $10^{-9}$. After the sensitivity receiver, Bessel low-pass filter (LPF) with 3-dB bandwidth of 7.5 GHz was used to reduce noise in the received signal. The BER estimator, the electric scope, as well as the optical spectrum analyser were used to study information obtained about the data and the quality of the propagated signal.

\section{RESULTS AND DISCUSSION}

While performing simulation, all of the above parameters were abiding, but optimal parameters such as CW power and HNLF length were defined experimentally. Results were analysed by the obtained spectrum of the optical signal at the HNLF output, the signal spectrum after the AWG multiplexer and the spectrum after the transmission line (where SMF span length was $20 \mathrm{~km}$ ). The quality of the transmitted signal was evaluated for the worst channel of the DWDM-
PON transmission system based on the eye diagram and BER results (where $\mathrm{BER} \leq 10^{-}$ ${ }^{10}$ ). The worst channel for a 4-channel and 16-channel DWDM system was the fourth channel, but for an 8-channel system, it was the first channel.

Using the optimal parameters as defined in Table 1, different spectra were obtained for FWM optical effect for up to 16-channel DWDM-PON transmission system (see Fig. 5) with data channels.

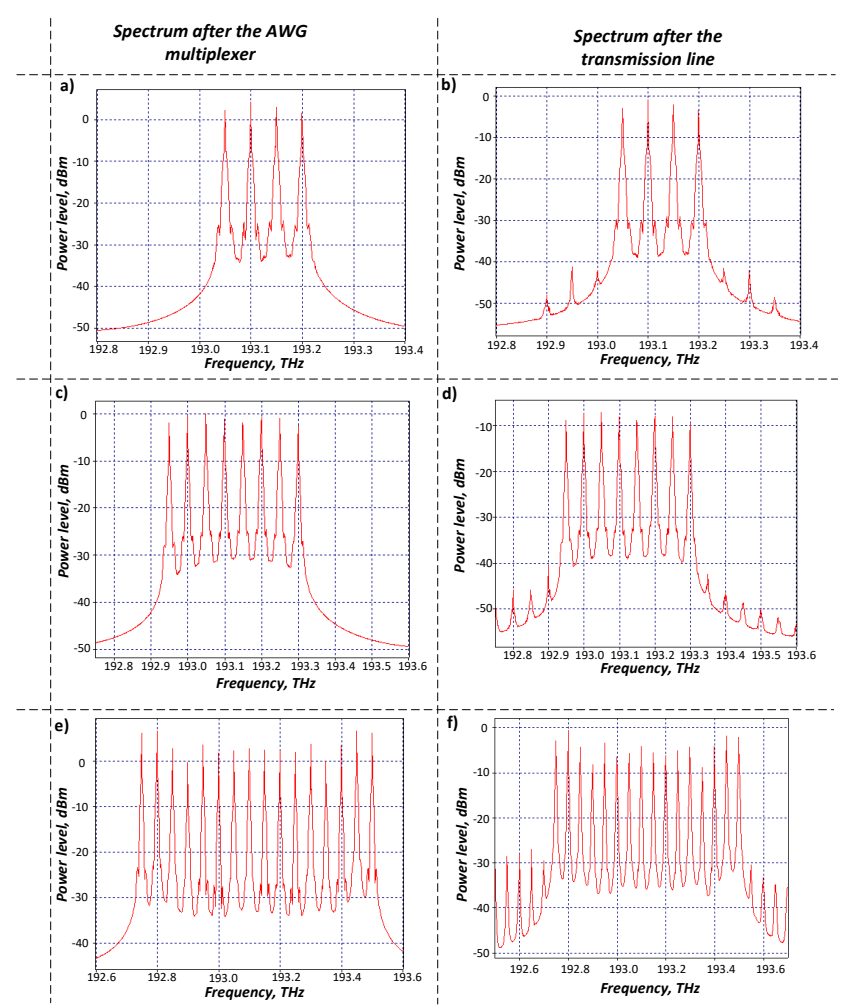

Fig. 5. The output spectrum of the DWDM-PON transmission system with FWM multi-wavelength source (a) 4-channel, (c) 8-channel, (e) 16-channel after the AWG multiplexer, and (b) 4-channel, (d) 8-channel, (f) 16-channel after the $20 \mathrm{~km}$ long fibre optical transmission line. 
Comparing the optical spectrum at the output of HNLF and AWG MUX, we can conclude that the channel output levels after AWG DEMUX/MUX are evenly distributed (Fig. 3 (a, c, e) generated harmonics before demultiplexing). In Fig. 5 (b, c, e) depending on the number of channels, we can see that in this case the demultiplexer separated 4,8 and 16 channels at frequencies from 192.75 to $193.5 \mathrm{THz}$ with a channel spacing of $50 \mathrm{GHz}$. The average calculated channel peak power output for 4 channels was $3.3 \mathrm{dBm}$. The largest difference between the calculated average channel peak power and the measured channel power levels was in the fourth channel, which was 0.5 $\mathrm{dBm}$. However, in an 8-channel system the average channel peak power was $0.7 \mathrm{dBm}$ where the difference between the worst channel was $1.3 \mathrm{dBm}$ (for the first channel). However, in a 16-channel DWDM-PON transmission system, the average channel peak power was $2.8 \mathrm{dBm}$, and the difference between the calculated average channel peak power and the measured channel peak power was $2.9 \mathrm{dBm}$ (for the fourth channel). The obtained values are acceptable because they fall within the $3 \mathrm{dBm}$ range. Figure 5 shows the spectrum for the DWDM-PON transmission system with (a) 4-channel, (c) 8-channel, (e) 16-channel after the AWG multiplexer, (b) 4-channel, (d) 8-channel, (f) 16-channel after the transmission line.

The quality of the following eye diagrams was evaluated (up to a 16-channel DWDM-PON system with transmission length of $20 \mathrm{~km}$ and the bitrate of $10 \mathrm{Gbit} / \mathrm{s}$ ) using the electrical scope. Figure 6 shows eye diagrams of the received signal in the (a) 4-channel, (b) 8-channel and (c)16channel DWDM-PON transmission system with an FWM multi-wavelength source.
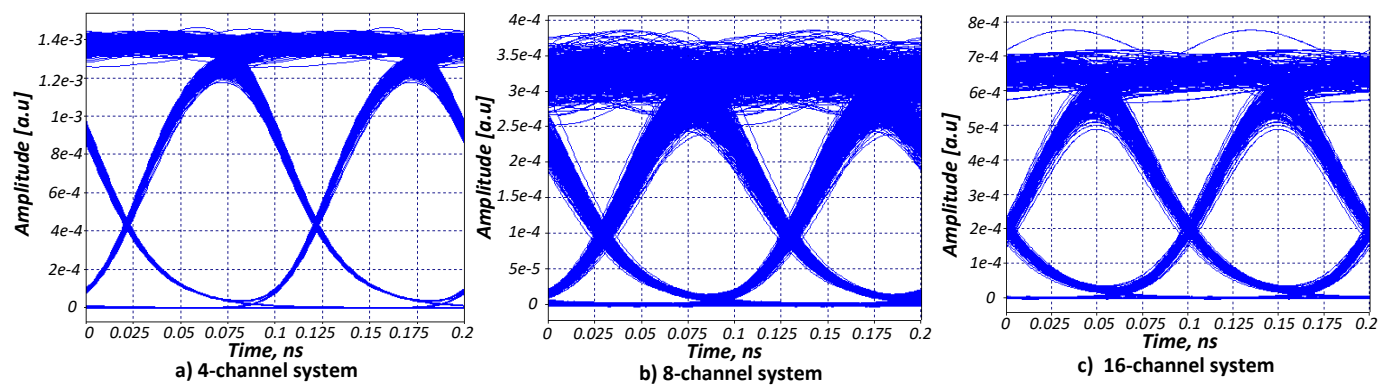

Fig. 6. Eye diagrams of the DWDM-PON transmission system with an FWM multi-wavelength source for (a) 4-channel, (b) 8-channel and (c) 16-channel.

As we can see from Fig. 6, the quality of the eye diagram decreases due to the increased dispersion and nonlinear effects (like FWM, SBS), and as more channels should be generated, the higher power fluctuations and therefore the overall performance of the system are lower. Table 2 shows BER values for up to 16-channel of the DWDM-PON transmission system, where the worst channel for a 4-channel and a 16-channel system is the fourth chan- nel, but for an 8-channel system - the first channel. However, it is considered that the transmission lines will perform qualitatively with low losses because the "eye" opening is wide in all cases and the BER is below the threshold. According to ITU-T G.984.2 related to the recommended BER value for fibre optical transmission systems with a bitrate of $10 \mathrm{Gbit} / \mathrm{s}$ per channel, BER should be less than $10^{-10}$ [16]. 
Table 2. Summary of BER Values of the Received Signals in up to 16-Channel DWDM-PON Transmission System after $20 \mathrm{~km}$ Transmission through SMF Fibre Span

\begin{tabular}{|l|c|c|c|}
\hline & 4-channel system & 8-channel system & 16-channel system \\
\hline $\begin{array}{l}\text { BER value for the worst } \\
\text { performing system channel }\end{array}$ & $1 \times{ }^{10-} 40$ & ${ }^{2} .5 \times 10-23$ & $6.5 \times{ }^{10-} 14$ \\
\hline
\end{tabular}

\section{CONCLUSIONS}

Within the framework of research, we investigated up to 16-channel DWDM-PON transmission system with an FWM multiwavelength source. FWM effect was used to generate a multi-carrier (multi-wavelength) light source for 4-, 8- and 16-channel DWDM-PON transmission system. The first step was to find the optimal power of CW pump lasers and HNLF fibre length for FWM carrier generation, which could be further used for modulation and transmission of data. It was found that for the simulated DWDM-PON transmission system with $10 \mathrm{Gbit} / \mathrm{s}$ bitrate and $50 \mathrm{GHz}$ channel spacing, the CW pumping power level for both lasers in the case of a 4-channel system was $+20 \mathrm{dBm}$ and the HNLF fibre length was $0.9 \mathrm{~km}$. To increase the number of DWDM-PON system channels from 4 channels to 8 and 16 channels and, accordingly, to generate more carriers, it was necessary to increase the power of pump CW lasers. Thus, for an 8-channel system, CW power was $24.1 \mathrm{dBm}$, and the length of HNLF was $1.39 \mathrm{~km}$, but for a 16-channel system it was $26.3 \mathrm{dBm}$ and $1.05 \mathrm{~km}$, respectively. The SBS threshold must be taken into account to increase the number of FWM generated carriers for the DWDM-PON system with 8 to 16 channels; otherwise, the carrier output power is uneven and, as a result, a 16-channel system cannot be created. Therefore, for the generation of 16 carriers and SBS suppression, we used additional phase modulation. BER values and eye diagrams were evaluated for the worst channel of the DWDM-PON system. Through the analysis of the received signal after $20 \mathrm{~km}$ transmission, it was observed that in a 4-channel DWDM system the BER value of the worstperforming channel was $1 \times 10^{-40}$. Nevertheless, in an 8-channel system, the BER value for the worst-performing channel was $2.5 \times 10^{-23}$, but in a 16 -channel system, the BER value of the worst-performing channel was $6.5 \times 10^{-14}$.

\section{ACKNOWLEDGEMENTS}

This work has been supported by the project "Generation of Kerr Combs in a Nonlinear Microresonator and Its Applications in Fiber Optical Communication Systems"
No. MP-2019/4, for strengthening scientific personnel capacity in the year 2019/2020 at Riga Technical University.

\section{REFERENCES}

1. Bobrovs, V., Porins, J. \& Ivanovs, G. (2007). Influence of Nonlinear Optical Effects on the NRZ and RZ Modulation.
Electronics and Electronica Engineering Signal Technology, 4 (76), 55-58. 
2. Olonkins, S., Spolitis, S., Lyashuk, I., \& Bobrovs, V. (2014). Cost effective WDMAON with multicarrier source based on dual-pump FOPA. In: 6th International Congress on Ultra Modern Telecommunications and Control Systems and Workshops (ICUMT), (pp. 23-28), 6-8 October 2014, St. Petersburg, Russia.

3. Gómez, F., Puerto, K., \& Guevara, D. (2015). Effect of nonlinear four-wave mixing in optical fibre transmission. In: 2015 IEEE Thirty Fifth Central American and Panama Convention (CONCAPAN $X X X V)$, (pp. 1-6), 11-13 November 2015, Tegucigalpa, Honduras.

4. Dilendorfs, V., Spolitis, S., \& Bobrovs, V. (2017). Effectiveness Evaluation of Dispersion Compensation Methods for Fiber-Optical Transmission Systems. Progress in Electromagnetic Research Symposium (PIERS), 3759-3763.

5. Selvamani, A., \& Sabapathi, T. (2011). Suppression of four wave mixing by optical phase conjugation in DWDM fiber optic link. In: International Conference on Recent Advancements in Electrical, Electronics and Control Engineering (pp. 95-99), 1517 December 2011, Sivakasi, India.

6. Sugumaran, S., \& Arulmozhivarman, P. (2013). Effect of chromatic dispersion on four-wave mixing in WDM systems and its suppression. In: International Conference on Emerging Trends in VLSI, Embedded System, Nano Electronics and Telecommunication System (ICEVENT) (pp. 1-5), 7-9 January 2013, India.

7. Cheng,M.C., Tsai,C.T.,Chi,Y.C., \&Lin,G.R. (2014). Direct QAM-OFDM Encoding of an L-band Master-to-Slave InjectionLocked WRC-FPLD Pair for $28 \times 20 \mathrm{~Gb} / \mathrm{s}$ DWDM-PON Transmission. IEEE Journals \& Magazines, 32 (17), 15724-15736.

8. Kachhatiya, V., \& Prince, S. (2016). Wavelength division multiplexing-dense wavelength division multiplexed passive optical network (WDM-DWDM-PON) for long reach terrain connectivity. In: InternationalConferenceon Communication and Signal Processing (ICCSP) (p. 1), 6-8 April 2016, Madras, India.
9. Telecommunication Standardization Sector of ITU. (2012). Spectral grids for WDM applications: DWDM frequency grid. Recommendation ITU-T G.694.1.

10. Spolitis S., Bobrovs, V., Berezins, S., \& Ivanovs, G. (2013). Optimal design of spectrally sliced ASE seeded WDMPON system. In: 2012 15th International Telecommunications Network Strategy and Planning Symposium (NETWORKS) (pp. 1-5), February 2013. Rome, Italy.

11. OFS Specialty Photonics Division. (2013). Highly Non-Linear Fiber (HNLF). Product Sheet, Version: 20131210, Denmark, 1, 2013.

12. Cerqueira Arismar, Jr. S., Chavez Boggio, J. M., Hernandez-Figueroa, H. E., Fragnito, H. L., \& Knight, J. C. (2008). Highly efficient generation of cascaded four-wave mixing products in a hybrid photonic crystal fiber. In: European Conference on Optical Communications - ECOC (pp. 16-20), 1620 September 2007, Berlin, Germany.

13. Kartalopoulos, S. V. (2008). Next generation intelligent optical networks. USA: Springer Science Business Media.

14. Agrawal, G. (2002). Fiber-optic communication systems (3rd ed.). New York: John Wiley \& Sons.

15. Dakin, J. P., \& Brown, R. G. W. (2018). Handbook of optoelectronics: Enabling technologies (Volume Two). USA: CRC Press Taylor \& Francis Group.

16. Telecommunication Standardization Sector of ITU. (2003). Gigabit-Capable Passive Optical Networks (GPON): Physical Media Dependent (PMD) Layer Specification. Recommendation ITU-T G.984.2. 